\title{
VALIDEZ Y CONFIABILIDAD DEL CUESTIONARIO DE INTERACCIÓN TRABAJO-FAMILIA (SWING) EN TRABAJADORES DE UNA EMPRESA MINERA DE LA LIBERTAD, PERÚ
}

\section{VALIDITY AND RELIABILITY OF THE WORK-FAMILY INTERACTION QUESTIONNAIRE ( SWING) IN WORKERS OF A MINING COMPANY OF LA LIBERTAD, PERÚ}

\author{
Nataly Briggete INGUNZA LASTRA ${ }^{1}$ \\ Psicóloga Organizacional de ADECCO \\ CONSULTING S.A.C
}

\section{Miguel Alfredo CARRASCO MUÑOZ²}

Director de la Unidad de Investigación de la Facultad de Psicología, Universidad Nacional Hermilio Valdizán. Perú.

$1 \quad$ Psicóloga Organizacional de ADECCO CONSULTING S.A.C y consultora de Evaluaciones de Riesgo Psicosocial. Perú.

Correo: ingunza.lastra@gmail.com

Orcid: 0000-0003-1520-536X

$2 \quad$ Catedrático principal de la Facultad de Psicología y Director de la Unidad de Investigación de la Facultad de Psicología de la Universidad Nacional Hermilio Valdizán. Huánuco-Perú.

Correo: miguelcm.29@gmail.com

Orcid: 0000-0002-4919-901X

\section{RESUMEN}

La presente investigación es de tipo psicométrico, descriptivo y correlacional del cuestionario español «Survey Work-Home Interaction-Nijmegen» (SWING), adaptado por Bernardo Moreno Jiménez para evaluar las relaciones entre el trabajo y la familia. Se 
estudiaron sus propiedades psicométricas a una muestra de 343 trabajadores, de una empresa minera de la Libertad, Perú. El proceso de validación del cuestionario comprendió en el análisis de la validez de contenido por criterio de jueces a través del coeficiente $V$ de Aiken. La evaluación de la características psicométricas se llevó a cabo en dos fases: en la primera con una muestra piloto para determinar el análisis de reactivos a través del índice de discriminación (la correlación ítem-test). La segunda fase con una muestra objetivo se determinó nuevamente el índice de la discriminación donde se modificó un ítem. Los resultados de los análisis de criterio confirmatorio obtenidos a través del gol estándar (entrevista semiestructuradas), nos indica una correlación media de Pearson con 0,579 . El análisis de la fiabilidad de la escala señaló que esta versión adaptada y validada para trabajadores mineros peruanos posee una buena consistencia interna, con valores que se situaron entre 0,825 y 0,892 por área. Se concluye que esta versión posee propiedades psicométricas adecuadas, y se sugieren pautas para el uso de la versión peruana de este cuestionario en futuras investigaciones. También se desarrolló una versión de software del cuestionario; el cual facilita la aplicación y corrección del cuestionario.

Palabras clave: validez de criterio, análisis de confiabilidad, trabajadores, psicometría, cuestionario.

\section{ABSTRACT}

The present research is of a psychometric, descriptive and correlational type of the Spanish questionnaire «Survey Work-Home InteractionNijmegen» (SWING), adapted by Bernardo Moreno Jiménez to evaluate the relations between work and family. Their psychometric properties were studied in a sample of 343 workers from a mining company of LibertadPerú 2016. The process of validation of the questionnaire comprised in the analysis of the validity of content by judges criterion through the coefficient $V$ of Aiken. The evaluation of the psychometric characteristics was carried out in two phases: in the first with a pilot sample to determine the analysis of reagents through the index of discrimination (the item-test correlation). The second phase with a target sample was again determined the index of discrimination where an item was modified. The results of the confirmatory criterion analysis make the four-factor model the one that best fits the data (obtained in the standard goal through the interviews). The analysis of reliability of the scale indicated that this adapted version and validity for Peruvian mining workers has a good internal consistency, with values ranging between 0.825 and 0.892 per area. It is concluded that this version has adequate psychometric properties, and suggested guidelines for the use of the Peruvian version of this questionnaire in future research. A software version of the questionnaire was also developed which facilitates the application and correction of the questionnaire.

Keywords: criterion validity, reliability analysis, workers, psychometry, questionnaire.

\section{INTRODUCCIÓN}

El trabajo es una de las actividades de mayor importancia para la integración del ajuste psicológico y social de los individuos de una sociedad. La Organización Internacional del Trabajo señala que tener un trabajo decente implica contar con oportunidades de un trabajo productivo, con seguridad en el lugar de trabajo y protección social para las familias, mejores perspectivas de desarrollo personal e integración a la sociedad, libertad para que la gente exprese sus opiniones, organización y participación en las decisiones que afectan sus vidas e igualdad de oportunidad y trato para todas las mujeres y hombres (Lewandowski, 2015), es decir, condiciones de trabajo que le permitan a la persona desarrollarse y tener un trabajo decente; es por ello que es importante 
conocer si existe una interacción positiva o negativa entre el trabajo y la familia. Los antecedentes sobre este tema se han centrado en examinar especialmente el conflicto existente entre estas dos esferas. El conflicto trabajofamilia tiene como consecuencia ser fuente de estrés, ya que existe una línea de investigación en la cual se evidencia entre los estresores y diversas consecuencias (También parece que falta algo) (Schneewind, María, \& Vita, 2004) en las personas que trabajan. De hecho, algunos estresores, como el conflicto o la ambigüedad de rol, no se relacionaban directamente con mayores niveles de tensión, estatus de salud física y mental (Jiménez, Isabel; Vergel; Rodríguez \& Geurts, 2009), depresión, hipertensión, pobre salud física, desórdenes de ansiedad, humor y abusos de sustancias; también está muy asociado a la insatisfacción laboral, bajo involucramiento en el trabajo y deterioro en el rendimiento (Morelos, 2012). En concreto, Greenhaus y Beutell (1985) se refirieron al término "conflicto trabajo-familia», definiéndolo como «una forma de conflicto de rol, en el que las presiones que resultan del trabajo y las familiares son mutuamente incompatibles en algún aspecto». A partir de esta definición, diversos autores señalaron la necesidad de explorar ambas direcciones del conflicto, con el fin de identificar si es el trabajo lo que dificulta el desempeño de las responsabilidades familiares (conflicto trabajo-familia) o viceversa (conflicto familia-trabajo) (Frone, Russell y Cooper, 1992).

En el Perú no existen instrumentos específicos para medir las relaciones entre el trabajo y la familia, lo que obstaculiza examinar las relaciones negativas y positivas entre ambos. Por todo lo expuesto, para el presente estudio se han formulado la siguiente pregunta de investigación:

¿Cuál es el grado de validez y confiabilidad del Cuestionario de Interacción Trabajo-Familia (SWING) en trabajadores de una empresa minera en La Libertad?
El objetivo de la investigación es determinar la validez, confiabilidad y baremos en puntajes percentilares del Cuestionario de Interacción Trabajo-Familia (SWING) en trabajadores de una empresa minera en La Libertad. La validación del cuestionario de interacción trabajo-familia y el planteamiento de alternativas de solución, hacen posible esclarecer los aportes de la Psicología Ocupacional, por intermedio de los profesionales que apelan a la teoría psicológica para la resolución de conflictos organizacionales. Finalmente, permite evidenciar la participación de la disciplina psicológica y su acumulado teórico en la implementación de los modelos, es decir, cómo se toma el producido de teoría en salud ocupacional en contextos de salud en las organizaciones.

\section{MATERIAL Y MÉTODOS}

La investigación es de tipo psicométrico descriptivo, ya que pretende describir la validez y confiabilidad del Cuestionario de Interacción Trabajo-Familia (SWING) y de análisis correlacional. Se realizó un muestreo probabilístico y la extracción de la muestra se realizó por el aleatorio estratificado (Hernández Sampieri et al., 2010) con afijación proporcional de las 38 contratas y compañía que conforman dicha empresa minera. La muestra está constituida por 343 trabajadores mineros de las distintas contratas y compañía. Los instrumentos empleados fueron el Cuestionario de Interacción Trabajo-Familia (SWING), la historia psicológica ocupacional y una ficha sociodemográfica.

Cuestionario de Interacción Trabajo-Familia. La validación de este instrumento se llevó a cabo según los pasos que recomienda la literatura sobre adaptación de tests y escalas (Hambleton, 1994; Hambleton, Merenda y Spielberger, 2005; Muñiz y Bartram, 2007). La primera fase del proceso consistió en la adaptación de la escala al Perú a través de un equipo de investigadores expertos en la materia sobre salud ocupacional. Los expertos que analizaron 
la formulación de cada uno de los ítems y reformularon algunos de ellos según criterios consensuados. En esta fase se contó también con el apoyo y colaboración del Dr. Bernardo Moreno Jiménez de la Universidad Autónoma de Madrid, quien desarrolló el SWING en España, con el fin de comprobar si se había comprendido correctamente el sentido de varios de los ítems; con ello se comprobó la equivalencia entre ambas versiones (Brislin, 1970). Las diferencias encontradas se resolvieron mediante discusión, llegando a una versión final consensuada.

Entrevista semiestructurada. Una de las técnicas de uso más frecuente que permite recabar información es la entrevista; se hace uso de esta cuando el tema de estudio es difícil de observar. El trabajo con una entrevista semiestructurada consiste en que el entrevistador posee una guía de preguntas, sin embargo se encuentra en la libertad de introducir preguntas adicionales en caso desee obtener mayor información sobre el tema (Hernández Sampieri et al., 2010)

Para la presente investigación se trabajó con una guía de entrevista teniendo en consideración los lineamientos teóricos que enmarcan el Conflicto Trabajo Familia. La entrevista recabó información acerca de la evaluación del conflicto trabajo-familia, que tanto positiva como negativamente impacta a la familia, además de las concepciones que tienen sobre cómo debería ser el funcionamiento familiar. Las preguntas en la guía de entrevista o Historia Psicológica Ocupacional fueron realizadas teniendo en consideración la GEMO002 "GUÍA DE EVALUACIÓN PSICOLÓGICA OCUPACIONAL", del Centro Nacional de Salud Ocupacional y Protección del Medio Ambiente; principalmente la historia permitió evaluar los cuatro aspectos del conflicto trabajo-familia. Además, se tomaron algunas preguntas adicionales por parte de los entrevistadores y se realizaron algunas modificaciones a estas, teniendo en cuenta nuestro objetivo. La propuesta de entrevista fue revisada en dos oportunidades por una psicóloga capacitada y con experiencia en salud ocupacional, de esta manera se obtuvo la versión final. Para el análisis de los datos se emplearon el programa estadístico SPSS18, Microsoft Excel 2010 y el Mintap.

\section{RESULTADOS}

En primer lugar, se analizaron los estadísticos descriptivos de los ítems, en concreto la media, la mediana, la desviación típica, así como el error típico. La validez de criterio del SWING se comprobó mediante análisis correlacional con el método de la correlación de Pearson y la $\boldsymbol{t}$ de Student. Los estadísticos descriptivos de media, mediana, desviación típica, para cada ítem, se muestran en la tabla 1. En ella podemos observar cómo las medias más altas se corresponden con los ítems pertenecientes a las dos subescalas de interacción positiva. Se puede observar que el ítem 18, perteneciente a la subescala de interacción negativa trabajo-familia, es el que presenta un valor mayor $(M=1,94)$. Sin embargo, las subescalas de interacción positiva presentan medias más bajas en general, siendo el ítem 6 el de menor puntuación $(M=1,01)$. 
Tabla 1.

Estadísticos descriptivos de los ítems $(\mathrm{N}=343)$

\begin{tabular}{|c|l|l|l|l|c|}
\hline & Media & Mediana & Ind. Disc & Varianza & Alfa \\
& & & & & Cronbach \\
\hline Ítem 01 & 1,61 & 1 & 0,556 & 0,309 & 0,810 \\
\hline Ítem 02 & 1,76 & 2 & 0,950 & 0,902 & 0,817 \\
\hline Ítem 03 & 1,24 & 0 & 1,092 & 1,193 & 0,810 \\
\hline Ítem 04 & 1,83 & 0 & 0,987 & 0,973 & 0,806 \\
\hline Ítem 05 & 1,83 & 0 & 0,915 & 0,837 & 0,808 \\
\hline Ítem 06 & 1,01 & 2 & 1,060 & 1,123 & 0,808 \\
\hline Ítem 07 & 1,73 & 2 & 0,978 & 0,956 & 0,811 \\
\hline Ítem 08 & 1,36 & 1 & 1,085 & 1,178 & 0,813 \\
\hline Ítem 09 & 1,27 & 1 & 0,517 & 0,267 & 0,807 \\
\hline Ítem 10 & 1,59 & 1 & 0,681 & 0,464 & 0,811 \\
\hline Ítem 11 & 1,64 & 1 & 0,767 & 0,589 & 0,812 \\
\hline Ítem 12 & 1,78 & 1 & 0,704 & 0,496 & 0,809 \\
\hline Ítem 13 & 1,60 & 1 & 1,406 & 1,977 & 0,819 \\
\hline Ítem 14 & 1,05 & 1 & 1,125 & 1,266 & 0,796 \\
\hline Ítem 15 & 1,94 & 2 & 1,207 & 1,458 & 0,791 \\
\hline Ítem 16 & 1,08 & 2 & 1,177 & 1,386 & 0,795 \\
\hline Ítem 17 & 1,25 & 2 & 1,142 & 1,304 & 0,800 \\
\hline Ítem 18 & 1,92 & 1 & 1,166 & 1,359 & 0,798 \\
\hline Ítem 19 & 1,73 & 1 & 1,078 & 1,163 & 0,800 \\
\hline Ítem 20 & 1,69 & 2 & 0,978 & 0,957 & 0,795 \\
\hline Ítem 21 & 1,87 & 2 & 1,042 & 1,087 & 0,792 \\
\hline Ítem 22 & 1,69 & 0 & 1,076 & 1,157 & 0,793 \\
\hline
\end{tabular}

Se analizó el índice de discriminación la correlación Ítem-Test (índice de discriminación); no se eliminó ningún ítem, pero sí se modificaron los ítems $1,2,13,17,20$ y 22 . En este análisis se concluyó que ningún ítem se encuentra con valores inferiores a 0,20 o valores negativos, por lo tanto, no se debe eliminar ningún ítem. (Merino, C., y Livia, 2009). Análisis de la validez de contenido interno

Se midió mediante una evaluación de cada ítem, hecha por 10 psicólogos especialistas en psicología ocupacional y psicometría, quienes juzgaron si cada uno de los ítems y dominios miden el concepto de interacción trabajo-familia. Primero, se evaluó la adaptación cultural, cuyo objetivo es comprobar si los ítems de la prueba miden el mismo constructo en una cultura distinta. Segundo, se realizó el análisis de la $V$ de Aiken; de un total de 22 ítems fueron considerados como significativos con el área que se pretende medir y evaluar, tanto en pertinencia del área y claridad de rol, obteniéndose una $\mathrm{V}$ de Aiken $=0,871$ para el área de interacción positiva trabajo-familia, una $V$ de Aiken $=0,906$ para el área de interacción positiva familia-trabajo, una $V$ de Aiken $=0,905$ para la área de interacción negativa trabajo-familia, una $\vee$ de Aiken $=0,905$ 
para el área de interacción negativa trabajofamilia. Siendo la $V$ de Aiken total de 0,89, en consideración a que se utilizó un intervalo de significancia riguroso de $\mathrm{Vo}=0,60$; teniendo en cuenta que es posible hacer el análisis en un nivel con mayor rigurosidad, como $\mathrm{Vo}=0,70$ (Merino, C., y Livia, 2009). Los intervalos de
Aiken también permiten hacer una estimación de número de jueces necesitados para lograr un grado de precisión del coeficiente de $\mathrm{V}$ de Aiken (Aliaga Tovar, J. 2006). Se consideraron como ítems significativos o todos ítems mayores a 0,5 (rango mínimo estándar preestablecido $\mathrm{V} \_0=$ $0.5)$ con un nivel de confianza del $5 \%(0,05)$. Obsérvese la tabla 2.

\begin{tabular}{|c|c|c|c|}
\hline Estadísticos des & $\begin{array}{l}\text { Tabla } 2 \\
\text { os de la }\end{array}$ & Aiken de los & ítems \\
\hline \multicolumn{4}{|c|}{$(\mathrm{N}=343)$} \\
\hline ÁREA DE LA PRUEBA & ITEMS & $\begin{array}{l}V \text {-AIKEN } \\
\text { POR ITEM }\end{array}$ & $\begin{array}{c}V \text {-AIKEN POR } \\
\text { AREA }\end{array}$ \\
\hline \multirow{8}{*}{$\begin{array}{l}\text { INTERACCIÓN POSITIVA } \\
\text { TRABAJO-FAMILIA }\end{array}$} & Ítem 01 & 0,775 & \multirow{8}{*}{0,871875} \\
\hline & Ítem 02 & 0,9 & \\
\hline & Ítem 03 & 1 & \\
\hline & Ítem 04 & 0,75 & \\
\hline & Ítem 05 & 1 & \\
\hline & Ítem 06 & 0,75 & \\
\hline & Ítem 07 & 0,9 & \\
\hline & Ítem 08 & 0,9 & \\
\hline \multirow{4}{*}{$\begin{array}{l}\text { INTERACCIÓN POSITIVA } \\
\text { FAMILIA-TRABAJO }\end{array}$} & Ítem 09 & 0,825 & \multirow[t]{4}{*}{0,90625} \\
\hline & Ítem 10 & 0,875 & \\
\hline & Ítem 11 & 1 & \\
\hline & Ítem 12 & 0,925 & \\
\hline \multirow{5}{*}{$\begin{array}{l}\text { INTERACCIÓN NEGATIVA } \\
\text { TRABAJO-FAMILIA }\end{array}$} & Ítem 13 & 0,8 & \multirow[t]{5}{*}{0,905} \\
\hline & Ítem 14 & 0,85 & \\
\hline & Ítem 15 & 0,975 & \\
\hline & Ítem 16 & 0,925 & \\
\hline & Ítem 17 & 0,975 & \\
\hline \multirow{5}{*}{$\begin{array}{l}\text { INTERACCIÓN NEGATIVA } \\
\text { FAMILIA-TRABAJO }\end{array}$} & Ítem 18 & 0,95 & \multirow[t]{5}{*}{0,905} \\
\hline & Ítem 19 & 1 & \\
\hline & Ítem 20 & 0,95 & \\
\hline & Ítem 21 & 0,8 & \\
\hline & Ítem 22 & 0,825 & \\
\hline V-AIKEN TOTAL & \multicolumn{3}{|c|}{0,893181818} \\
\hline
\end{tabular}

Análisis de la validez de criterio externa 
La correlación de Pearson con 0,579 nos indica una correlación media, previamente establecido el $5 \%(0,05)$ de margen de error inferior al 0,01. Pero es necesario establecer la significancia de la correlación para determinar finalmente el coeficiente de validación de criterio. Se transformó la correlación ( $r$ ), en $\boldsymbol{t}$ de student mediante la fórmula planteada, obteniendo como resultado una $t$ de student transformada de $\boldsymbol{t}=11,60$. La $\boldsymbol{t}$ de student transformada $(11,60)$ se ubica dentro del área de control preestablecido en el nivel de confianza (95\%) y el margen de error $(0,05)$ con 320 grados de libertad $(\mathrm{N}$ 2). Es decir, la correlación es estadísticamente significativa al nivel del 0,05 de margen de error.

\section{ANÁLISIS DE LA CONFIABILIDAD}

También se examinó la fiabilidad de las cuatro dimensiones del cuestionario calculando su consistencia interna mediante el índice alfa de Cronbach. Como puede observarse en la tabla 1 , los valores obtenidos se situaron entre 0,79 y 0,81 , por encima del 0,80 que califica como bueno recomendado (Nunnally y Berstein, 1994).

\section{DISCUSIÓN}

De acuerdo a los objetivos planteados para esta investigación, se determinó la validez y confiabilidad y normalización del Cuestionario de Interacción Trabajo-Familia (SWING), aplicado a una muestra de trabajadores mineros de La Libertad. Los resultados encontrados en el análisis psicométrico aportan evidencia a favor de la construcción del Cuestionario de Interacción Trabajo-Familia (SWING), obteniéndose niveles de validez y confiabilidad aceptables, por lo tanto la prueba puede aplicarse a una población que comparta las mismas características que la población objetivo. Los procedimientos estadísticos que se utilizaron son: la validez de contenido, a través del índice $\checkmark$ de Aiken; la validez de criterio, a través del gol estándar; el análisis de ítems, a través del índice de discriminación; la consistencia interna, a través del coeficiente alfa de Cronbach; la estandarización, a través de los puntajes totales o percentiles; el punto de corte, a través de la curva de ROC. Los resultados observados nos indican que el proceso de validación del Cuestionario de Interacción Trabajo-Familia (Swing) es adecuado a los sujetos de la muestra, es por ello que se realizaron dos procesos de validez, tal como lo hicieron Sergio Alexis Domínguez Lara en el análisis psicométrico de la escala de cansancio emocional en estudiantes de una universidad privada (2013). La importancia del presente trabajo de investigación se centra en poder brindar a la comunidad científica un instrumento validado en trabajadores mineros que permita estudiar con mayor certeza el conflicto trabajo-familia. Como aseguran Jiménez et al., 2009, la importancia de estudiar el conflicto trabajo-familia se debe a que en todas las organizaciones no hay una atención al tema de la familia y cómo este interfiere en el trabajo. Ello debido a que los riesgos psicosociales de doble presencia o conflicto trabajo-familia son parte de la evaluación en salud ocupacional a nivel mundial, ya que está asociado a depresión, hipertensión, pobre salud física, desórdenes de ansiedad, humor y abusos de sustancias; también está muy asociado a la insatisfacción laboral, bajo involucramiento en el trabajo y deterioro en el rendimiento. Por lo tanto, al conocer si un trabajador tiene el conflicto trabajo-familia de interacción positiva o negativa, ayuda a la organización a mejorar las condiciones en el aspecto de los riesgos psicosociales. A continuación, se discutirán los hallazgos referidos a la confiabilidad y validez del Cuestionario de Interacción Trabajo-Familia (Swing) aplicado en trabajadores mineros. Esto se realizará a la luz de la revisión teórica, las adaptaciones previas y las características de la muestra, considerando tanto los atributos demográficos como las variables relacionadas al tipo de trabajo que realizan en la mina. El primer objetivo que tuvo esta investigación fue realizar la adaptación de la escala, teniendo en cuenta 
las características de nuestra población con el objetivo de lograr trasmitir el significado original. Si bien los ítems no presentaron problemas para ser entendidos por los grupos focales, durante la aplicación del piloto los participantes formularon preguntas a la investigadora respecto al significado que tomaban los ítems redactados en doble negación cuando se marcaban las opciones nunca, a veces, a menudo o siempre. Debido a ello, y a sugerencia del estadístico, se incrementó una opción de pregunta más entendible y de mayor aplicabilidad estadística a nunca, muy pocas veces, muchas veces, casi siempre y siempre. Esta dificultad se dio independientemente del grado de instrucción.

Al analizar las características psicométricas del Cuestionario de Interacción Trabajo-Familia (Swing), primero se determinó la confiabilidad del instrumento, encontrándose una consistencia interna elevada, lo cual indica que los resultados obtenidos son consistentes y no se deben al azar. Ya en investigaciones anteriores se mantuvo una estructura similar al trabajo original de Geurts et al (2005), donde se ha evaluado la confiabilidad del instrumento con el mismo estadístico, tales como la adaptación francesa realizada por Lourel et al. (2005), estudios que obtuvieron coeficientes de confiabilidad. Todas las escalas superan el criterio de 0,70 recomendado (Nunally y Berstein, 1994; Dikkers, Geurts, Kompier, Taris, Houtman y van den Heuvel, 2007; Taris, Verhoeven, Geurts, Kompier y van der Linden, 2006; Dikkers et al., 2007). Además, el nivel de confiabilidad obtenido en esta investigación cumple con los criterios requeridos en comparación a las investigaciones previamente señaladas, por lo que se puede afirmar que el instrumento está midiendo, de manera consistente, el constructo para los trabajadores mineros que pertenecen a un nivel socioeconómico medio-alto de La Libertad.

Al analizar la confiabilidad de cada una de las dimensiones se obtuvo el alfa de Cronbach de las dimensiones, que osciló entre 0,70 y 0,87 . Se encontró que las cuatro dimensiones presentaban una confiabilidad aceptable que componen el Cuestionario de Interacción Trabajo-Familia (Swing). Estos resultados son similares a los valores obtenidos en la versión original.

\section{REFERENCIAS BIBLIOGRÁFICAS}

Aiken, L. R., Yang, W., Soto, M., Segovia, L., Binomial, P., Miller, J. M., Moreno, R. (2011). Diseño y validación de un cuestionario para analizar la calidad en empleados de servicios deportivos públicos de las mancomunidades de municipios extremeñas. Educational and Psychological Measurement. http://doi. org/10.1177/0013164412473825

Aliaga Tovar, J. (2006). «Psicometria: Tests psicométricos, confiabilidad y validez». Psicología: Tópicos de Actualidad, 85-108. Retrieved from http://uigv.edu.pe/facultades/psicologia/ recursos/05LibroEAPAliaga.pdf

Dikkers, J., Geurts, S., Kompier, M., Taris, T., Houtman, I., y van den Heuvel, F. (2007). «Does workload cause work-home interference or is it the other way around? "Stress and Health: Journal of the International Society for the Investigation of Stress, 23(5), 303-314.

Frone, M. R., Russell, M., \& Cooper, M. L. (2003). Work-family balance (American $P)$. Washington.

Frone, M. R., Russel, M., \& Cooper, M. L. (1992). «Prevalence of Work-Family Conflict: Are work and family boundaries asymmetrically permeable? " Journal of Organizational Behavior, 13(7), 723-729. http://doi.org/10.1002/job.4030130708

Geurts, S., Taris, T.W., Kompier, M.A.J., Dikkers, 
J.S.E., V. H., \& M.L.M., y Kinnunen, U. M. (n.d.). Work-home interaction from a work psychological perspective: Development and validation of a new questionnaire, the SWING. Work \& Stress.

Grzywacz, J.G. (2000). «Work-family spillover and health during midlife: Is managing conflict everything? » American Journal of Health Promotion, 14, 236-243.

Hernandez Sampieri, R., Fernandez Collado, C., \& Baptista Lucio, M. del P. (2010). Metodología de la investigación. Retrieved from http://www.casadellibro.com/librometodologia-de-la-investigacion-5-edincluye-cd-rom/9786071502919/1960006

Jiménez, B. M., Isabel, A., Vergel, S., Rodríguez, A., \& Geurts, S. (2009). Propiedades psicométricas de la versión española del Cuestionario de Interacción TrabajoFamilia (SWING), 21, 331-337.

Hambleton, R.K., Merenda, P.F., y Spielberger, C.D. (2005). Adapting educational and psychological tests for cross-cultural assessment. London: Lawrence Erlbaum Associates.

Kinnunen U., Feldt T, Geurts S, P. L. (2006). Types of Work Family Interface WellBeing Correlates of Negative and Positive Spillover Between Work and Family. Scand J Psychol., 47, 149-162.

Lewandowski, C. M. (2015). The Effects of Brief Mindfulness Intervention on Acute Pain Experience: An Examination of Individual Difference. http://doi.org/10.1017/ CBO9781107415324.004

Merino, C., y Livia, J. (2009). Intervalos de confianza asimétricos para el índice de validez de contenido: un programa Visual Basic para la $V$ de Aiken. (Psicothema, Ed.) (Psicothema).

Morelos, C. (2012). Propiedades Psicométricas del Cuestionario de Interacción TrabajoFamilia NijmeGen (SWING) en Empleados de.

Morelos, J. (2012). Propiedades psicométricas del Cuestionario de Interacción TrabajoFamilia NijmeGen (SWING) en empleados de Cuernavaca, Morelos, México (PDF Download Available). Available from: https://www.researchgate.net/ publication/233778239_Propiedades_ Psicometricas_del_Cuestionario_ de_Interaccion_Trabajo-Familia_ NijmeGen_SWING_en_Empleados_de_ Cuernavaca_Morelos_Mexico [accessed Sep 11, 2017].

Nunnally, J.C., y Berstein, I.H. (1994). Psychometric theory (3 ${ }^{\text {rd }}$ ed.). New York, NY: McGraw-Hill; 1995

Organización Mundial de la Salud. (2003). La Familia y la Salud, 23-27. Retrieved from http://www1.paho.org/spanish/gov/ce/ ce132-14-s.pdf

Schneewind, K. A., Maria, A., \& Vita, D. (2004). «Interested in European research? EU RESEARCH ON SOCIAL SCIENCES AND HUMANITIES Family Life and Professional Work: Conflict and Synergy (FamWork)» A State-of-the-Art Report. Retrieved from http://europa.eu.int/comm/ research/rtdinfo/index_en.html 